\title{
Imaging Live Cell Dynamics using Snapshot Hyperspectral Image Mapping Spectrometry
}

Amicia D. Elliott ${ }^{1}$, Noah Bedard ${ }^{2}$, Alessandro Ustione ${ }^{1}$, Michelle A. Baird ${ }^{3}$, Michael W.

Davidson $^{3}$, Tomasz S. Tkaczyk ${ }^{2}$, and David W. Piston ${ }^{1}$

${ }^{1}$ Vanderbilt University Medical Center, Nashville, TN, USA.

${ }^{2}$ Rice University, Houston, TX, USA.

${ }^{3}$ The Florida State University, Tallahassee, Florida, USA.

Spectral imaging techniques are useful for a number of biological experiments, with particular importance in live-cell imaging of fluorescent proteins. Hyperspectral imaging methods collect the whole spectrum per pixel in an image, allowing for the use of multiple probes in a single experiment. Often, these probes are fluorescent protein-based biosensors that rely on either intramolecular Forster resonance energy transfer (FRET), or conjugation of circularly-permuted fluorescent proteins to a conformationally-sensitive peptide. While the increasing number of available biosensors makes this methodology attractive for studying multiple molecular processes simultaneously, the narrow spectral range of fluorescent proteins limit its ultimate usefulness.

Many FRET-based biosensors are composed of a cyan fluorescent protein (CFP) variant donor and a yellow fluorescent protein (YFP) variant acceptor. While there are a few green/red FRET pairs, dual-FRET is rarely attempted due to the overlapping emission spectra of all four fluorophores and challenges in configuring a single excitation with a snapshot modality, required for simultaneous imaging. A further challenge lies in data analysis, as the quantitative value of FRET experiments relies upon reference spectra, high spectral resolution, and high quality data. Finally, most multispectral imaging systems use scanning, or require increased illumination to achieve a high enough SNR across the spectrum. These methods are limited in spectral or temporal resolution and have an insufficient dynamic range for quantifying the small changes associated with most FRET-based biosensors.

An approach that overcomes many of these limitations utilizes the Image Mapping Spectrometer (IMS), a snapshot hyperspectral imaging system, which couples to a light microscope and rapidly collects high-resolution spectral data in a single snapshot. It functions by spatially distributing neighboring pixels to create blank regions into which spectrally dispersed wavelength content can be mapped onto a 2D detector array. This method allows parallel measurement of a sample's $x, y, \lambda$ datacube without scanning, thus collecting the whole datacube in a single snapshot with high optical throughput. 
We have previously demonstrated that the IMS coupled to a widefield microscope is useful for simultaneously studying cAMP and $\mathrm{Ca}^{2+}$ signaling dynamics in pancreatic $\beta$-cells [1]. The biosensors in that study included a cyan/yellow cAMP FRET sensor $\left({ }^{\mathrm{T}}\right.$-Epac- $\left.{ }^{\mathrm{VV}}\right)$ and a cpGFPbased $\mathrm{Ca}^{2+}$ sensor. To further develop the capabilities of the IMS approach, we prepared a Caspase-3 cleavable biosensor (GRScat) with the recently published green/red FRET pair mClover and mRuby2 [2], to be used concurrently with the ${ }^{\mathrm{T}}$-Epac- ${ }^{\mathrm{VV}}$ FRET sensor (Figure 1). Here, we utilize these sensors in a dual FRET approach with the IMS to understand the relationship between cAMP signaling and Caspase-3-mediated apoptosis in pancreatic $\beta$-cells,
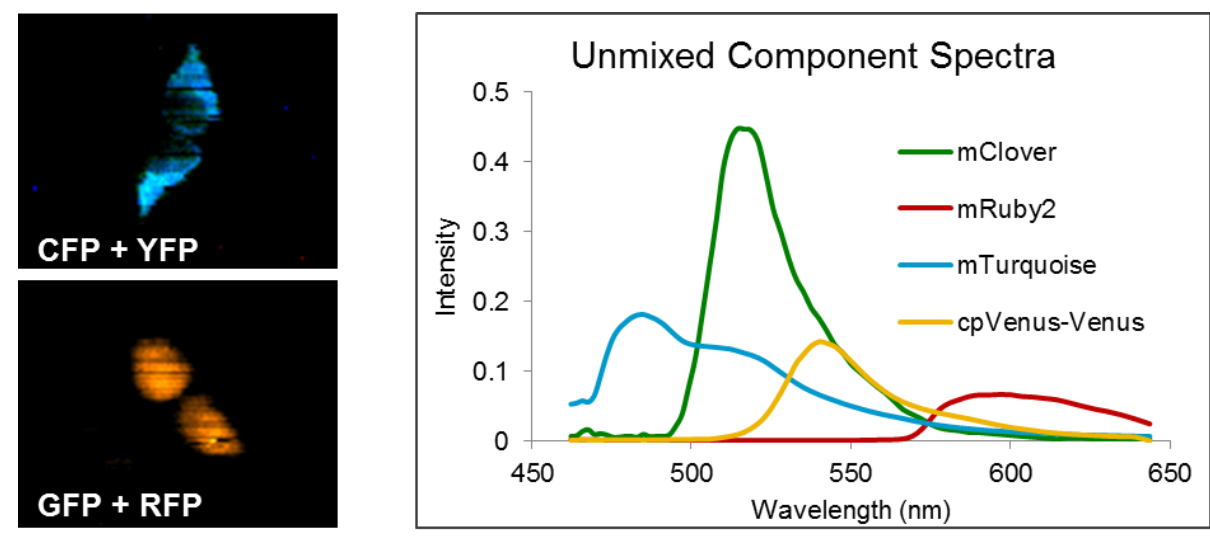

Figure 1. $\beta$-cells transfected with the ${ }^{\mathrm{T}}$-Epac- ${ }^{\mathrm{VV}}$ FRET sensor (top left), GRScat FRET sensor (bottom left), and the unmixed spectra of a cell expressing both biosensors. which plays an important role in the pathology of type 2 diabetes. In many cell types, oxidative stress has been show to contribute to apoptotic cell death, but the molecular mechanism is less well understood. It has been shown that treatment of $\beta$-cells with hydrogen peroxide to induce oxidative damage increases apoptosis. However, preincubation with forskolin, a cAMP activator, protected against cell death. These experiments were conducted using traditional biochemistry, which provides only a single snapshot (via Western blot) of a dynamic process [3]. Here we have used the IMS approach and treated cells with hydrogen peroxide to interrogate the relationship between these signaling pathways, and identify the time course of their activity in an effort to better understand a potential way to protect against oxidative stress-induced cell death.

\section{References}

[1] Elliott, et al, J. Cell Sci. 125 (20), 4833-4840 (2012).

[2] Lam, et al, Nat. Methods. 9, 1005-1012 (2012).

[3] Van de Velde, et al, PNAS. 108 (41), 16876-16882 (2011). 